\title{
INCIDENCIAS DE LAS TECNOLOGÍAS DE LA INFORMACIÒN Y COMUNICACIÒN EN EL PROCESO ENSEÑANZA - APRENDIZAJE
}

\author{
AUTORES: Karla Maribel Quiñonez Paucar ${ }^{1}$ \\ Helen Lisseth Bone Quiñonez ${ }^{2}$ \\ Pascual Ángel Pisco Gómez ${ }^{3}$
}

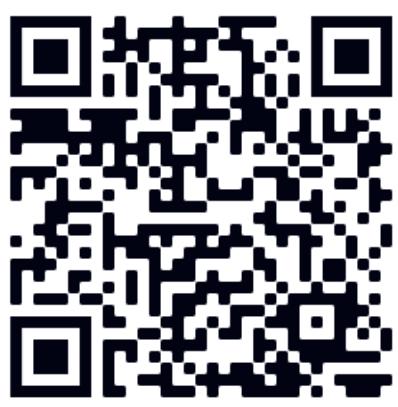

DIRECCIÓN PARA CORRESPONDENCIA:karlacore14@gmail.com

Fecha de recepción: 03/05/2019

Fecha de aceptación: 09/07/2019

\section{RESUMEN}

En el presente trabajo se da a conocer el diseño, desarrollo y el proceso de enseñanza aprendizaje que están a la par con los avances en el campo del diseño instructivo y de las teorías. Las bases que tradicionalmente se han manejado desde la Tecnología Educativa, deben ser sustituidas por otras que se ajusten mejor a un nuevo paradigma centrado en el aprendizaje. Pero si bien es cierto que necesitamos nuevos fundamentos, también que el nuevo paradigma necesita incorporar la mayoría del conocimiento generado por las teorías previas de diseño instructivo, aunque este conocimiento necesite ser reestructurado sustancialmente para que pueda adaptarse a las nuevas necesidades educativas de la Unidad Educativa María Piedad del Castillo de Levi del cantón Paján. El objetivo de este artículo tiene como finalidad diseñar un nuevo instructivo del proceso de enseñanza aprendizaje con nuevas tecnologías educativas la cual de un aporte positivo al estudiante de esta Institución Educativa la cual carece de Tecnologías de La Información y comunicación. La metodología que lleva el estudio de este tema es totalmente investigativa de la mano con los encargados de esta Respetable Institución. La Unidad Educativa María Piedad Castillo de Levi del Cantón Pajan, apunta hacia un mejor modelo de diseño instructivo, para brindarles mejores conocimientos a sus docentes como a sus estudiantes, a través de nuevas tecnologías impulsadas por los conocimientos dados del día a día de las nuevas investigaciones que se generan en el mundo entero.

PALABRAS CLAVE: impulsadas, paradigma, metodología, instructivos.

\section{THE IMPACT OF ICT ON TEACHING ACADEMIC}

\footnotetext{
1 Karla Maribel Quiñonez Paucar - Estudiante de Octavo semestre de la Carrera de Ingeniería en Sistemas Computacionales - Universidad Estatal del Sur de Manabí Jipijapa-Manabí-Ecuador- karlacore14@gmail.com

2 elen Lisseth Bone Quiñonez - Estudiante de Octavo semestre de la Carrera de Ingeniería en Sistemas Computacionales - Universidad Estatal del Sur de Manabí Jipijapa-Manabí-Ecuador.

${ }^{3}$ Ing. Ángel Pisco Gómez - Master en Gerencia Educativa - Docente de Carrera Tecnologías de la Información en la Universidad Estatal del Sur de Manabí Jipijapa-Manabí-Ecuador.
} 
Karla Maribel Quiñonez Paucar, Helen Lisseth Bone Quiñonez, Pascual Ángel Pisco Gómez...

\section{ABSTRACT}

In this Scientific Article we present the design, development and teaching-learning process that are on a par with the advances in the field of instructional design and theories. The bases that have traditionally been handled from the Educational Technology, must be replaced by others that better fit a new paradigm focused on learning. But while it is true that we need new fundamentals, also that the new paradigm needs to incorporate most of the knowledge generated by the previous theories of instructive design, although this knowledge needs to be substantially restructured so that it can adapt to the new educational needs of the Educational Unit Maria Piedad from the Castillo de Levi in Paján canton. The objective of this article is to design a new instruction on the teaching-learning process with new educational technologies which provides a positive contribution to the student of this Educational Institution which lacks information and communication technologies. The methodology that leads the study of this topic is entirely investigative hand in hand with those responsible for this Respectable Institution. Maria Piedad Castillo de Levi Educational Unit of the Paján Canton, points towards a better instructional design model, to provide better knowledge to its teachers as well as to its students, through new technologies driven by the knowledge given of the day to day of the new investigations that are generated in the whole world.

KEYWORDS: driven, paradigm, methodology, instructive

\section{INTRODUCCIÓN}

Las bases desde donde abordar los procesos de diseño, desarrollo y evaluación de los procesos de enseñanza-aprendizaje están íntimamente relacionadas con los avances en el campo del diseño instructivo y de las teorías, concepciones y modelos relacionados con dicho diseño: teorías del aprendizaje, teorías del currículo, de la instrucción, de la comunicación, el desarrollo de los sistemas de información, Las bases que tradicionalmente se han manejado desde la Tecnología Educativa, parece que deben ser sustituidas por otras que se ajusten mejor a un nuevo paradigma centrado en el aprendizaje. Pero si bien es cierto que necesitamos nuevos fundamentos, también lo es que el nuevo paradigma necesita incorporar la mayoría del conocimiento generado por las teorías previas de diseño instructivo, aunque este conocimiento necesite ser reestructurado sustancialmente para que pueda adaptarse a las nuevas necesidades educativas, (Jesús Salinas Ibañez, 2007).

El concepto de diseño instructivo, aun en sus acepciones más amplias, incluye no sólo el proceso de planificación del marco instructivo, del sistema de distribución, etc., sino también, y fundamentalmente, la planificación de los materiales didácticos, lo que ha sido visto así desde los inicios de la Tecnología Educativa, (Briggs, Romiszowski, Reigeluth, \& Merrill, 2007).

La Unidad Educativa María Piedad Castillo de Levi del Cantón Pajan, apunta hacia un mejor modelo de diseño instructivo, para brindarles mejores conocimientos a sus docentes como a sus estudiantes, a través de nuevas tecnologías impulsadas por los conocimientos dados del día a día de las nuevas investigaciones que se generan en el mundo entero.

Estos conocimientos educativos impartidos por la Tecnología de la Información y Comunicación, son beneficios a la tecnología de los futuros emprendimientos que va a tener esta unidad educativa del cantón Paján provincia de Manabí, teniendo en cuenta que nuevos diseños de procesos de enseñanzas van hacer de un nivel muy superior a lo anteriormente impartido en dicha Unidad Educativa. 
Las TIC, busca mejorar las enseñanzas y subir de niveles los conocimientos de ciertas unidades académicas alrededor del mundo, este va a ser el caso de la Unidad Educativa María Piedad Castillo de Levi del Cantón Pajan, que tiene falencias de aprendizajes no muy deficientes, pero si un porcentaje mayor a lo normal con respecto a la educación.

La integración de las TIC en el sistema educativo conlleva dificultades, entre ellas la insuficiente preparación del profesorado para introducirlas en su práctica habitual de aula (Fernández-Cruz y Fernández-Díaz, 2016). Las escuelas no disponen de un proyecto consensuado en relación a la utilización de las TIC y, en consecuencia, muchas de las prácticas didácticas que se llevan a cabo con las tecnologías digitales, no representan una verdadera innovación o mejora con respecto a las prácticas tradicionales de enseñanza (Christian y Mathrani, 2014). Por tanto, la falta de conocimientos y actualización del profesorado, tanto sobre el uso de las TIC, desde un punto de vista puramente instrumental, como sobre las actividades innovadoras basadas en las mismas, desde una perspectiva más metodológica, dificultan una buena implementación de éstas en el contexto educativo. Abundando en este planteamiento García-Valcárcel y Tejedor (2010), identifican algunos factores que dificultan la integración y utilización de las TIC en los procesos de enseñanza-aprendizaje, los cuales se exponen a continuación (p. 127):

- Ineficaz formación del profesorado para el uso de tecnología adecuada a las necesidades de los alumnos. Formación de tipo instrumental, fragmentada, individualista y ajena a las necesidades de una escuela concreta.

- Escaso tiempo disponible de los profesores para la colaboración entre ellos y el desarrollo de programas de tecnología integrada.

- Carencia de personal disponible para mantener los ordenadores y solucionar problemas técnicos y de aplicación didáctica (coordinador / responsable TIC).

- Falta de ordenadores y accesibilidad a Internet en todas las aulas (no sólo en laboratorios o aulas específicas).

Es lógico que, la integración de las TIC en el sistema educativo, ha soportado dificultades y limitaciones. Y referente, se han realizados diferentes esfuerzos muy considerables dados por políticas tanto de carácter nacional como autonómico, entre otras iniciativas previas, lo que ha generado permuta y transformaciones en la enseñanza dentro del sistema educativo. No hay que olvidar que nuestro Sistema de Educación se va mejorando día a día hecho de que en Ecuador y todos sus alrededores presenta precisamente la peculiaridad de no contar con un plan común, bien coordinado y consensuado a nivel estatal. (Área, 2010, 2006). La descripción de algunos rasgos de las políticas educativas dirigidas a la integración de las TIC a nivel europeo, nacional y autonómico pone en evidencia la necesidad de caracterizar las buenas prácticas docentes, como un eje vertebrador de la innovación educativa y del modelo a seguir (De Pablos, 2010).

Finalmente, con estas nuevas tecnologías y nuevos modelos de aprendizajes se va a lograr mejorar las condiciones de enseñanzas de esta unidad Educativa e impartir como ejemplo a otras unidades educativas que tengan estas incidencias de (Tecnología de la Información y Comunicación).

\section{DESARROLLO}

Diseño de procesos de enseñanzas-aprendizajes mediante TIC y diseño instructivos para la "Unidad Educativa María Piedad Castillo de Levi del Cantón Paján". 
Funciones a considerar para el diseño de enseñanza y aprendizajes mediante las TIC en la "Unidad Educativa María Piedad Castillo de Levi del Cantón Paján".

\section{METODOLOGÍA}

Posibilidades de interacción - Trabajo colaborativo - Multicultural.

\section{MULTIMEDIA}

Capacidad multimedia requerida - Capacidad multimedia disponible.

\section{EQUIPOS Y RED}

Vidas previsibles de los equipos.

Capacidad técnica.

Características de las redes.

\section{FUNCIONES A CUBRIR}

Tutorial, transmisión de contenidos.

Trabajo colaborativo.

Creación de materiales.

\section{SINCRONIA Y ASINCRONOMIA}

Capacidad sincrónica de las herramientas.

Capacidad asincrónica de las herramientas.

\section{LAS HERRAMIENTAS}

Manejabilidad.

Adaptabilidad.

Accesibilidad.

Navegabilidad.

Versatilidad.

Tipo de código (abierto o cerrado).

\section{EL DOCENTE}

Habilidades no directivas.

Formación metodológica.

Habilidades sociales para la colaboración.

\section{EL ALUMNO}

4 UNESUM-Ciencias. Publicación cuatrimestral. Vol. 3, Año 2019, No. 2 (Mayo - Agosto) 
Autonomía.

Responsabilidad.

Conocimientos previos.

Investigador.

Actividades sociales para la colaboración.

Seguridad.

\section{DIFERENCIAS ENTRE LAS HERRAMIENTAS TECNOLÓGICAS Y OTROS RECURSOS.}

Las tecnologías basadas en entornos interactivos tienen algunas cualidades primordiales, que los diferencian de los medios típicos estáticos, que a largo plazo tendrán un impacto en la educación. Anteriormente hemos mencionado algunas de estos atributos como la ejecutabilidad de las representaciones. Los cambios significativos que la tecnología está logrando tienen que ver con tres características propias de estos recursos:

- La facilidad de tener a la mano diversas representaciones de un mismo concepto matemático o de lenguaje y poder relacionarlas activamente unas con otras.

- La "manipulación" de objetos matemáticos y sus relaciones.

La interactividad diferencia enormemente el recurso computacional de otros medios estáticos y aún dinámicos como los medios audiovisuales. Si se escribe una oración en un medio estático tal como el lápiz y el papel, ésta simplemente se imprime y no hay interactividad ni con el papel ni con otras frases que pudieran haber estado escritas antes en la hoja. Más aún, el papel no proporciona ayuda sobre lo que uno está escribiendo, salvo algunos casos en los que las cuadrículas o el papel para gráficas proporcionen algún soporte que guía a quién hace el dibujo. En forma semejante se puede ver la televisión y no hacer nada físico con ella a menos que se decida cambiar de canal o ajustar la imagen. En un sentido amplio podría decirse que todas las representaciones en todos los medios son interactivas, dado que el usuario hace una representación de lo que ve o lee. Pero este no es el sentido que queremos dar. Por medio interactivo vamos a considerar un medio que contribuye físicamente al sistema de representación que se pone en funcionamiento y hace que el estudiante se motive en aprender los saberes que el docente le imparte en el aula de clases, está comprobado científicamente que estos medios audiovisuales que se utilizan a diario a la par de la tecnología si aportan considerablemente con el estímulo de aprender por parte del estudiante que en este entorno jugaría un papel muy importante en el sistema educativo que se quiere mejorar y se está mejorando pero todo va de la mano con el estímulo de las TIC.

En el aula digital el estudiante se apropia de un contenido previamente seleccionado que responde a un sistema de objetivos lógicamente diseñados. Pero el carácter educativo de la enseñanza no sólo se queda en este plano, sino que va más allá al considerar también las influencias educativas que ejercen métodos, medios y las formas de evaluación empleadas en el aula digital. Forma también parte de este carácter educativo la interacción generada entre los estudiantes, ya sea por medio de un chat, un foro o el correo electrónico, por sólo citar algunas formas de interacción. El 
Karla Maribel Quiñonez Paucar, Helen Lisseth Bone Quiñonez, Pascual Ángel Pisco Gómez...

estudiante eleva su autonomía y responsabilidad ante su propio aprendizaje y contribuye, con su participación en el aula digital, al aprendizaje de los otros compañeros de estudio.

\section{UNIDAD DE LA INSTRUCCIÓN Y LA EDUCACIÓN.}

Hay que salir del error al considerar que en el aula digital los estudiantes sólo alcanzan un nivel instructivo que los pone en contacto con los contenidos, sin embargo, estos no están fuera de un contexto histórico y cultural. Lo anterior propone y te lleva a discernir que no es verdad que cualquier actividad dirigida a separar lo instructivo de lo educativo. La formación de la actividad cognoscitiva del individuo, la apropiación de conocimientos, el desarrollo del pensamiento teórico, de capacidades y habilidades intelectuales y profesionales, que tradicionalmente se consideran como resultado de la instrucción, constituyen también un producto fundamental de la educación. Es que la instrucción es una condición básica fundamental de la relación del ser humano con la naturaleza, con el medio y con las demás personas y de su actividad transformadora de la realidad que a su vez contiene, de forma indisoluble, aspectos éticos y emotivos.

\section{LIBERTAD DEL ESTUDIANTE Y SU RESPONSABILIDAD EN EL APRENDIZAJE}

El día a día que el estudiante se separa más de las formas tradicionales de enseñanza, es decir presenciales, y se sumerge en las actividades donde la presencia ligada no es el elemento más expresivo, aporta a su grado de libertad, autonomía ante el aprendizaje. Sus dudas, sus fallos deben ser resueltos a partir del estudio de otros materiales o de la controversia en foros o chat, donde se necesite, además, la objetividad y precisión de su planteamiento. Indiscutible para elevar esta autonomía se requiere que el diseño del aula digital contemple todo el elemento necesario para que el estudiante se vea beneficiado en su auto aprendizaje.

Con estos temas propuestos se pretende dar una idea significativa en la cual fomentar el sistema educativo de la Unidad Educativa María Piedad Castillo de Levi del Cantón Pajan. Y así dar una mejora a esta incidencia que se encuentra perenne en esta institución.

\section{MARCO TEÓRICO}

\section{Fundamentos desde la tecnología educativa:}

(Chadwick, 1979), recoge la contribución que estos referentes aportan a la T.E. Para este autor, el papel de la Psicología es el más relevante, al ser uno de sus intereses fundamentales el aprendizaje, objeto que comparte con aquélla. También se refiere a las contribuciones del enfoque sistémico desde donde se aportan herramientas, metodología y conceptos. Por su parte, el ámbito de la comunicación, si bien con un grado de influencia de menor envergadura -según Chadwick-, contribuirá desde el tratamiento de los medios.

Todas las herramientas que contribuyen al sistema de tecnología son muy importas ya que generan un mejor aporte para la sociedad académica y mejorar su aprendizaje.

\section{Diseño instructivo como proceso:}

(Reigeluth, 1999), remarca el carácter probabilístico de los métodos que surgen como diseño instructivo, al incrementar las opciones de alcanzar los objetivos en lugar de asegurar el logro de los mismos (determinismo).

El diseño instructivo es un medio de orientación y de guía académica y tecnológica, que ayuda al desarrollo superior de las unidades académicas estudiantiles para un futuro mejor en la sociedad. 


\section{Teoría general del sistema:}

(Pablos, 1994), la Teoría General de Sistemas, como propuesta integradora de la ciencia, aplicada a las situaciones educativas pretenderá "regular y controlar" la totalidad de variables que intervienen, describiendo en su totalidad el proceso, ofreciendo pautas de intervención.

Toda propuesta tiene que integrarse con el desarrollo de una investigación consolidada para un bienestar a la sociedad y que contribuya con el mayor cambio posible en lo que respecta a tecnología, medios de comunicación, a sistemas altos avances tecnológicos.

\section{Principios de diseño:}

Según (Romizowsky, 1997), probablemente sea debido a que el trabajo de diseño es tan complejo y tan dependiente del contexto que convierte en casi imposible el contraste y la prueba empírica de la usabilidad de un diseño respecto a otros. En consecuencia, seleccionar un modelo de diseño instructivo es un proceso racional, no empírico.

Los principios de diseños son factores de complejos que ayudan al desarrollo de una investigación integradora, ya sea para un mejor cambio a la calidad de vida de los estudiantes y seguir aportando las tecnologías hacia ello es gran cambio, pero con las debidas responsabilidades.

\section{Criterios de evaluación:}

Para (Salinas, 1992), existen otras modalidades para un mejor proceso de evaluación.

- Evaluación prospectiva o evaluación relativa al contexto (context evaluatíon). Tiene como fin ofrecer alternativas a la toma de decisiones concernientes al desarrollo de un proyecto (metas del programa, necesidades a satisfacer, etc. Se trata, en definitiva, de evaluar cuáles son los medios mejor adaptados a un determinado proyecto pedagógico, a un sistema educativo, a una situación, a unas condiciones.

- Las metas propuestas en proyectos tecnológicos, se dan con la intención de satisfacer las necesidades del cliente ya sea el caso. Pero a las unidades académicas hay que tomarlas desde otro punto estratégico para lograr llegar hacia ellas con base tecnológicas que se puedan integrar a su desarrollo académico.

\section{CONCLUSIONES}

Se pudo determinar que con la mejor Implementación de Tecnologías de la Información y Comunicación en la Unidad Educativa María Piedad del Castillo de Levi del cantón se podrá llevar un diseño de instructivo tecnológico donde van a tomar tecnologías educativas que motiven al estudiante para su mayor desempeño cuando el profesor imparta su clase dando un aporte positivo a la Institución y al aprendizaje académico del estudiante. Como bien lo hemos constatado anteriormente que esta Unidad Educativa hay un considerable porcentaje de falencias tecnológicas las cuales retrasan el nivel académico del estudiante con esta metodología investigativa hemos llegado a la conclusión de implementar un diseño instructivo donde lleve el itinerario de todos los procesos de enseñanza aprendizaje educativa a la par con la tecnología de la Información y comunicación aportando positivamente a la institución.

\section{REFERÉNCIAS BIBLIOGRÁFICAS}

Briggs, 1., Romiszowski, 1., Reigeluth, 1., \& Merrill, 1. (DOMINGO de DICIEMBRE de 2007). 5-libro-nuevastecnologc3adas-aplicadas-a-la-educacic3b3n-julio-cabero.pdf. Obtenido de 5-libro-nuevastecnologc3adas-aplicadas-a-la-educacic3b3n-julio-cabero.pdf: 
Karla Maribel Quiñonez Paucar, Helen Lisseth Bone Quiñonez, Pascual Ángel Pisco Gómez...

https://uogestiondelaprendizaje.files.wordpress.com/2015/03/5-libro-nuevas-tecnologc3adas-aplicadas-ala-educacic3b3n-julio-cabero.pdf

Chadwick. (lunes de Diciembre de 1979). 5-libro-nuevas-tecnologc3adas-aplicadas-a-la-educacic3b3n-juliocabero.pdf. Obtenido de 5-libro-nuevas-tecnologc3adas-aplicadas-a-la-educacic3b3n-julio-cabero.pdf: https://uogestiondelaprendizaje.files.wordpress.com/2015/03/5-libro-nuevas-tecnologc3adas-aplicadas-ala-educacic3b3n-julio-cabero.pdf

Jesús Salinas Ibañez, S. U. (DONMINGO de DICIEMBRE de 2007). 5-libro-nuevas-tecnologc3adas-aplicadas-a-laeducacic3b3n-julio-cabero.pdf. Obtenido de 5-libro-nuevas-tecnologc3adas-aplicadas-a-la-educacic $3 b 3 n$ julio-cabero.pdf: https://uogestiondelaprendizaje.files.wordpress.com/2015/03/5-libro-nuevastecnologc3adas-aplicadas-a-la-educacic3b3n-julio-cabero.pdf

Pablos, D. (1994). 5-libro-nuevas-tecnologc3adas-aplicadas-a-la-educacic3b3n-julio-cabero.pdf. Obtenido de 5libro-nuevas-tecnologc3adas-aplicadas-a-la-educacic 3 b3n-julio-cabero.pdf: https://uogestiondelaprendizaje.files.wordpress.com/2015/03/5-libro-nuevas-tecnologc3adas-aplicadas-ala-educacic3b3n-julio-cabero.pdf

Reigeluth. (lunes de diciembre de 1999). 5-libro-nuevas-tecnologc3adas-aplicadas-a-la-educacic3b3n-juliocabero.pdf. Obtenido de 5-libro-nuevas-tecnologc3adas-aplicadas-a-la-educacic3b3n-julio-cabero.pdf: https://uogestiondelaprendizaje.files.wordpress.com/2015/03/5-libro-nuevas-tecnologc3adas-aplicadas-ala-educacic3b3n-julio-cabero.pdf

Romizowsky, (. (1997). 5-libro-nuevas-tecnologc3adas-aplicadas-a-la-educacic3b3n-julio-cabero.pdf. Obtenido de 5-libro-nuevas-tecnologc3adas-aplicadas-a-la-educacic3b3n-julio-cabero.pdf: https://uogestiondelaprendizaje.files.wordpress.com/2015/03/5-libro-nuevas-tecnologc3adas-aplicadas-ala-educacic3b3n-julio-cabero.pdf

Salinas. (1992). 5-libro-nuevas-tecnologc3adas-aplicadas-a-la-educacic3b3n-julio-cabero.pdf. Obtenido de 5-libronuevas-tecnologc3adas-aplicadas-a-la-educacic3b3n-julio-cabero.pdf:

https://uogestiondelaprendizaje.files.wordpress.com/2015/03/5-libro-nuevas-tecnologc3adas-aplicadas-ala-educacic3b3n-julio-cabero.pdf 